\title{
DEVELOPMENT OF DRYING PROTOCOL FOR WITHANIA SOMNIFERA ROOTS
}

\author{
SONI ${ }^{1 *}$, HIMMAT SINGH CHAWRA ${ }^{1}$, SHARMA RK ${ }^{1}$, GARG R ${ }^{2}$
}

${ }^{1}$ Department of Pharmacy, School of Pharmacy, Suresh Gyan Vihar University, Jaipur, Rajasthan, India. ${ }^{2}$ Department of Pharmacy, B. R. Nahata College of Pharmacy, Mandsaur, Madhya Pradesh, India. Email: swetabrncp@gmail.com

Received: 10 July 2018, Revised and Accepted: 17 August 2018

ABSTRACT

Objective: This experiment was conducted to develop a drying protocol for Withania somnifera roots (during the year 2009-2010 under the National Medicinal Plants Board project, B.R. Nahata College of Pharmacy-Scientific Institute of Research Organization, Mandsaur [M.P.]).

Methods: The roots of Withania were harvested and cut into different sizes (2-4 inches) and subjected into sun drying, shade drying, and hot air oven drying in temperature ranging from $40^{\circ} \mathrm{C}$ to $60^{\circ} \mathrm{C}$. The temperature for sun drying ranging from $32^{\circ} \mathrm{C}$ to $42^{\circ} \mathrm{C}$ during daytime, and in night hours, the samples were kept in plastic covers to prevent reabsorption of moisture. In shade drying, the pieces of roots were kept in shade (proper air flowing place) at normal climatic temperature. Moreover, in hot air oven drying method/mechanical drying method, the pieces of roots were kept at $50^{\circ} \mathrm{C}$, $55^{\circ} \mathrm{C}$, and $60^{\circ} \mathrm{C}$, respectively. The samples were weighed, and the moisture content of the samples was taken at regular intervals until the standard moisture content of the dried roots was obtained that is mentioned in literature (10-5\%).

Result: The result revealed that hot air oven drying of roots took the lowest time $(12 \mathrm{~h})$, while sun drying method took $24 \mathrm{~h}$ for drying. The alkaloid content, namely, Withanolide A was significantly influenced by different methods of drying. The highest alkaloid content (0.010\%) was observed in sun drying and hot air oven drying with 0.021 .abc\% as compared to shade drying with $0.009 \%$ Withanolide A.

Conclusion: It may be concluded that sun drying and hot air oven drying are suitable methods for drying $W$. somnifera roots.

Keywords: Withania somnifera, Shade drying, Moisture content, Withanolide A.

(C) 2018 The Authors. Published by Innovare Academic Sciences Pvt Ltd. This is an open access article under the CC BY license (http://creativecommons. org/licenses/by/4. 0/) DOI: http://dx.doi.org/10.22159/ajpcr.2018.v11i10.28402

\section{INTRODUCTION}

Ashwagandha (Withania somnifera), also known as Indian ginseng, is an important ancient plant, and the roots of which have been employed in Indian traditional systems of Medicine, Ayurveda, and Unani. Ashwagandha belongs to family Solanaceae, and it is a green shrub [1] found throughout the drier parts of India, Baluchistan, Pakistan, Afghanistan, Sri Lanka, Congo, South Africa, Egypt, Morocco, and Jordan. In India, it is widely grown in the provinces of Madhya Pradesh, Uttar Pradesh, plains of Punjab, and northwestern parts of India such as Gujarat and Rajasthan [2].

Many pharmacological studies have been carried out to describe multiple biological properties of $W$. somnifera. These studies have shown that the plant preparation has antitumor, immunomodulatory, antispasmodic, tuberculosis, rheumatism, diuretic, antibiotic, adaptogenic, antioxidant activities, digestive disorders, and common cold [3].

\section{Constituents}

It is cultivated specifically for its root, which has medicinal and commercial value due to the presence of the steroidal chemicals, withanine and somniferine; therefore, it is considered the equivalent of Chinese ginseng (Kumar et al. 2008). The major constituents of $W$. somnifera are steroidal alkaloids and lactones, a class of constituents together known as withanolides (steroidal lactones with ergostane skeleton) [4]. So far 12 alkaloids, 35 withanolides, and several sitoindosides have been isolated, and their structures have been elucidated [5]. The various alkaloids include withanine, somniferine, somnine, somniferinine, withananine, pseudo-withanine, tropine, pseudo-tropine, 3- $\alpha$-gloyloxytropane, choline, cuscohygrine, isopelletirine, anaferine, and anahydrine [6].

It is all about the plant and the present article discusses the preservation technique/drying technique for roots of $W$. somnifera.
Drying

Drying is an essential process in the preservation of agricultural products. Drying consists of the removal of sufficient moisture content of crude drug so as to improve its quality and make it resistant to the growth of microorganisms. When medicinal plant materials are prepared for use in dry form, the moisture content of the material should be kept as low as possible to reduce damage from mold and other microbial infestation. Information on the appropriate moisture content for particular medicinal plant materials may be available from pharmacopeias or other authoritative monographs. A definition of drying that shall sharply differentiate it from evaporation is difficult to formulate. The term drying usually infers the removal of relatively small amounts of water from solid or nearly solid material, and the term evaporation is limited to the removal of relatively large amounts of water from solutions [4]. Drying inhibits partially enzymatic reactions. Drying facilitates pulverizing or grinding of a crude drug [7].

\section{METHODS}

An experiment was conducted to standardize the postharvest technology drying for $W$. somniferum during the year 2009-2010 at National Medicinal Plants Board Project, in B.R. Nahata College of Pharmacy, Mandsaur, M.P.

The drying studies were conducted using three different methods: Sun drying, shade drying, and hot air oven drying. Fresh ashwagandha roots were brought from the B.R. Nahata College of Pharmacy, Mandsaur (M.P.). The initial moisture content (60.4\%) of the Ashwagandha roots was noted. Then, these roots of plants cut in equal size 2-4 inches and kept for drying using different methods such as sun drying, shade drying, and hot air oven drying at the temperature range of $30-60^{\circ} \mathrm{C}$ (for roots) and the relative humidity range of 30-70\%. In sun drying method, the roots (2-4 inches) pieces were spread uniformly in the plastic trays and 
kept open to the sun from 8.00 am to $5.00 \mathrm{pm}$. Moreover, the moisture content of the dried material was taken every day using IR moisture balance. The temperature during sun drying ranged from $32^{\circ} \mathrm{C}$ to $42^{\circ} \mathrm{C}$ and during the night hours, and the samples were kept in plastic covers to prevent reabsorption of moisture. Likewise in mechanical/ hot air oven drying method, the root pieces (2-4 inches) were spread uniformly in the hot air oven at temperature $50^{\circ} \mathrm{C}, 60^{\circ} \mathrm{C}$, and $70^{\circ} \mathrm{C}$, respectively. Moreover, in shade drying method, the root pieces (2-4 inches) were spread in a plastic tray and kept in shade, well-ventilated place. The temperature during shade drying ranged from $25.5^{\circ} \mathrm{C}$ to $37^{\circ} \mathrm{C}$ and during the night hours, and samples were kept in plastic covers to prevent reabsorption of moisture. The samples were weighed at regular intervals until a moisture content of nearly $6-5 \%$ was obtained.

Table 1 shows that 6 days' sun drying is sufficient for the roots of ashwagandha. Moreover, moisture content limit for dried ashwagandha root is $6 \%$ or below $6 \%$ [10].
Table 2 shows that 15 days' shade drying is sufficient for roots of ashwagandha. The moisture content limit for dried ashwagandha root is $6 \%$ or below $6 \%$. Moreover, the experimental moisture content for dried roots of ashwagandha was $5.8 \%$ recorded.

Table 3 showing that when roots were kept in hot air oven for $12 \mathrm{~h}$ at $50^{\circ} \mathrm{C}$ and $60^{\circ} \mathrm{C}$ was sufficient for rapid drying of roots of Ashwagandha because it attained $6.2 \%$ and $5.8 \%$ moisture content at this time and temperature. Moreover, moisture content limit for dried ashwagandha root is $6 \%$ or below $6 \%$. Table 4 showing that hot air oven for $12 \mathrm{~h}$ at $50^{\circ} \mathrm{C}$ is sufficient for rapid drying of roots of Ashwagandha because it attains $6.0 \%$ moisture content at this time and temperature. Moreover, moisture content limit for dried ashwagandha root is $6 \%$ or below $6 \%$. Table 5 shows that hot air oven drying for $12 \mathrm{~h}$ at $60^{\circ} \mathrm{C}$ is sufficient for rapid drying of roots of Ashwagandha because it attains 5.8\% moisture content at this time and temperature. Moreover, moisture content limit for dried ashwagandha root is $6 \%$ or below $6 \%$. Table 6

Table 1: Sun drying of ashwagandha roots

\begin{tabular}{|c|c|c|c|c|c|}
\hline S. No. & Drying time (days) & Temperature $\left({ }^{\circ} \mathrm{C}\right)$ & RH (\%) & Weight of roots (g) & Moisture content (\%) \\
\hline 1. & 0 & 32.5 & 67 & 2000 & 60.4 \\
\hline 2. & 1 & $34.0-39.5$ & $43-25$ & 1500 & 50.4 \\
\hline 3. & 2 & $39.5-40.0$ & $30-26$ & 900.0 & 30.8 \\
\hline 4. & 3 & $36.0-39.5$ & $43-25$ & 800.5 & 24.4 \\
\hline 5. & 4 & $40.0-40.5$ & $40-34$ & 700.0 & 17.2 \\
\hline 6. & 5 & $38.5-39.0$ & $34-25$ & 600.5 & 10.4 \\
\hline 7. & 6 & $38.5-40.0$ & $38-26$ & 500.0 & 5.8 \\
\hline
\end{tabular}

Table 2: Shade drying of ashwagandha roots

\begin{tabular}{llllll}
\hline S. No. & Drying time (days) & Temperature $\left({ }^{\circ} \mathbf{C}\right)$ & RH (\%) & Weight of dried roots of WS (g) \\
\hline 1. & 0 & 36.5 & 67 & 2000.0 & Moisture content (\%) \\
2. & 1 & $36.0-34.0$ & $43-38$ & 1700.0 & 50.4 \\
3. & 2 & $37.0-36.0$ & $37-34$ & 1500.0 & 55.0 \\
4. & 3 & $36.5-34.0$ & $46-38$ & 1370.0 & 43.8 \\
5. & 4 & $37.5-36.0$ & $49-37$ & 1250.0 & 39.4 \\
6. & 5 & $36.5-35.5$ & $43-38$ & 1130.0 & 35.8 \\
7. & 6 & $37.5-36.0$ & $40-35$ & 1050.0 & 32.4 \\
8. & 7 & $37.0-36.5$ & $39-30$ & 985.0 & 28.2 \\
9. & 8 & $36.0-34.5$ & $35-30$ & 947.0 & 22.6 \\
10. & 9 & $37.5-36.0$ & $36-34$ & 939.0 & 20.2 \\
11. & 10 & $36.5-37.0$ & $34-30$ & 923.0 & 16.2 \\
12. & 11 & $36.0-37.5$ & $40-36$ & 905.0 & 8.0 \\
13. & 12 & $36.5-37.0$ & $43-38$ & 892.0 & 8.0 \\
14. & 13 & $34.5-36.0$ & $57-37$ & 885.0 \\
15. & 14 & $35.5-37.0$ & $46-38$ & 860.0 \\
16. & 15 & $36.0-37.5$ & $45-35$ & 545.0 \\
\hline
\end{tabular}

Table 3: Hot air oven drying of ashwagandha roots at constant time (12 h)

\begin{tabular}{|c|c|c|c|c|c|}
\hline S. No. & Drying time (h) & Temperature $\left({ }^{\circ} \mathrm{C}\right)$ & RH (\%) & Weight of dried roots of WS (g) & Moisture content (\%) \\
\hline 1. & Fresh & 37 & 40 & 1000.0 & 60.4 \\
\hline 2. & 12 & 50 & 37 & 480.5 & 6.2 \\
\hline 3. & 12 & 60 & 21 & 430.6 & 5.8 \\
\hline 4. & 12 & 70 & 14 & 410.0 & 5.2 \\
\hline
\end{tabular}

Drying time: Was kept constant $(12 \mathrm{~h})$. Temperatures: $50^{\circ} \mathrm{C}, 60^{\circ} \mathrm{C}$, and $70^{\circ} \mathrm{C}$ of hot air oven, $\mathrm{RH} \%$ : In hot air oven

Table 4: Hot air oven drying at constant temperature $\left(50^{\circ} \mathrm{C}\right)$

\begin{tabular}{|c|c|c|c|c|c|}
\hline S. No. & Drying time (h) & Temperature $\left({ }^{\circ} \mathrm{C}\right)$ & RH (\%) & Weight of dried roots of WS (g) & Moisture content (\%) \\
\hline 1. & Fresh & 37 & 58 & 1000.0 & 60.4 \\
\hline 2. & 6 & 50 & 78 & 700.5 & 19.4 \\
\hline 3. & 8 & 50 & 70 & 580.0 & 14.2 \\
\hline 4. & 10 & 50 & 58 & 480.5 & 6.8 \\
\hline 5. & 12 & 50 & 50 & 460.0 & 6.0 \\
\hline 6. & 14 & 50 & 47 & 455.6 & 5.2 \\
\hline
\end{tabular}


Table 5: Hot air oven drying at constant temperature $\left(60^{\circ} \mathrm{C}\right)$

\begin{tabular}{|c|c|c|c|c|c|}
\hline S. No. & Drying Time (h) & Temperature $\left({ }^{\circ} \mathrm{C}\right)$ & RH (\%) & Weight of dried roots of WS (g) & Moisture content (\%) \\
\hline 1. & Fresh & 37 & 58 & 1000.0 & 60.4 \\
\hline 2. & 6 & 60 & 76 & 680.7 & 17.4 \\
\hline 3. & 8 & 60 & 75 & 560.8 & 12.2 \\
\hline 4. & 10 & 60 & 50 & 450.0 & 6.4 \\
\hline 5. & 12 & 60 & 48 & 440.0 & 5.8 \\
\hline 6. & 14 & 60 & 45 & 435.0 & 4.4 \\
\hline
\end{tabular}

Table 6: Hot air oven drying at constant temperature $\left(70^{\circ} \mathrm{C}\right)$

\begin{tabular}{llllll}
\hline S. No. & Drying time $(\mathbf{h})$ & Temperature $\left({ }^{\circ} \mathbf{C}\right)$ & RH $(\%)$ & Weight of dried roots of WS (g) & Moisture content $(\%)$ \\
\hline 1. & Fresh & 37 & 58 & 1000.0 & 60.4 \\
2. & 6 & 70 & 76 & 640.7 & 15.4 \\
3. & 8 & 70 & 67 & 516.8 & 13.0 \\
4. & 10 & 70 & 52 & 410.0 & 5.8 \\
5. & 12 & 70 & 46 & 400.0 & 5.2 \\
6. & 14 & 70 & 43 & 398.0 & 4.0 \\
\hline
\end{tabular}

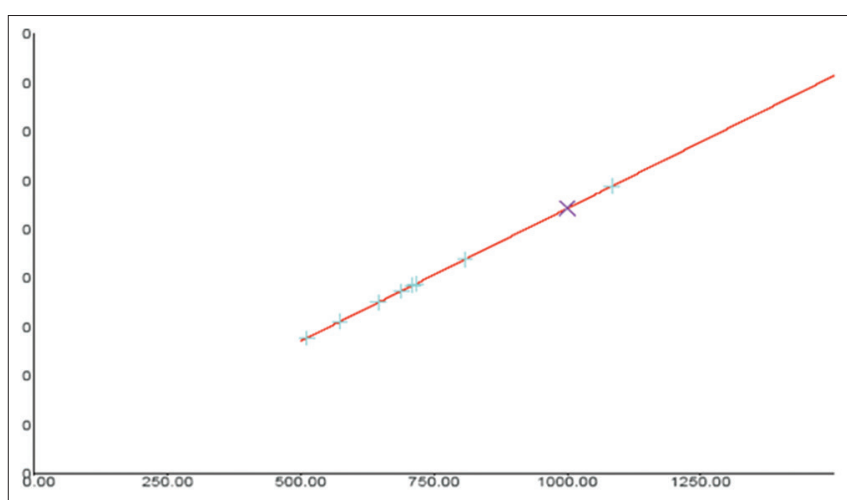

Fig. 1: Calibration curve for Withanolide A (regression through area)

showing that in hot air oven $10 \mathrm{~h}$ at $70^{\circ} \mathrm{C}$ is sufficient for rapid drying of roots of Ashwagandha because it was attaining 5.2\% moisture content at that time and temperature.. And moisture content limit for dried Ashwagandha root is $6 \%$ or below $6 \%$ [10].

Chemo profiling of dried roots of Ashwagandha

High-performance thin-layer chromatography (HPTLC) was performed for checking the chemical breakdown of ashwagandha roots during drying in which roots were dried by three different methods of drying. HPTLC method used as a analytical tool on the basis, of which the best method of drying for Ashwagandha roots was selected.

\section{Specifications}

CAMAG TLC scanner, stationary phase E. MERCK KGaA HPTLC plates silica gel $60 \mathrm{~F}_{254}$; dimensions $20.0 \mathrm{~cm} \times 10.0 \mathrm{~cm}$; drying device Oven temperature $120^{\circ} \mathrm{C}$ for $20 \mathrm{~min}$ (for plate); calibration mode was single level, and evaluation mode was peak area. Sample applicator was CAMAG Automatic TLC Sampler 4. The number of tracks was 14 in the form of bands (in which number of standard levels 1 and number of samples 13), band length was $8.0 \mathrm{~mm}$, and the distance between tracks was $12.3 \mathrm{~mm}$. Sample volume for application was $5.0 \mu \mathrm{l}$ for pure standard and $10 \mu \mathrm{l}$ for samples.

Development of TLC plates was done in Twin Trough Chamber $20 \mathrm{~cm}$ $\times 10 \mathrm{~cm}$ and saturated for $30 \mathrm{~min}$ with filter paper. The mobile phase was chloroform: methanol; drying device CAMAG TLC Plate Heater III temperature $60^{\circ} \mathrm{C}$ for $5 \mathrm{~min}$.

Derivatization post-chromatographic derivatization was done by Vanillin-Sulfuric acid reagent at CAMAG TLC plate heater III; temperature $120^{\circ} \mathrm{C}$ for $20 \mathrm{~min}$.

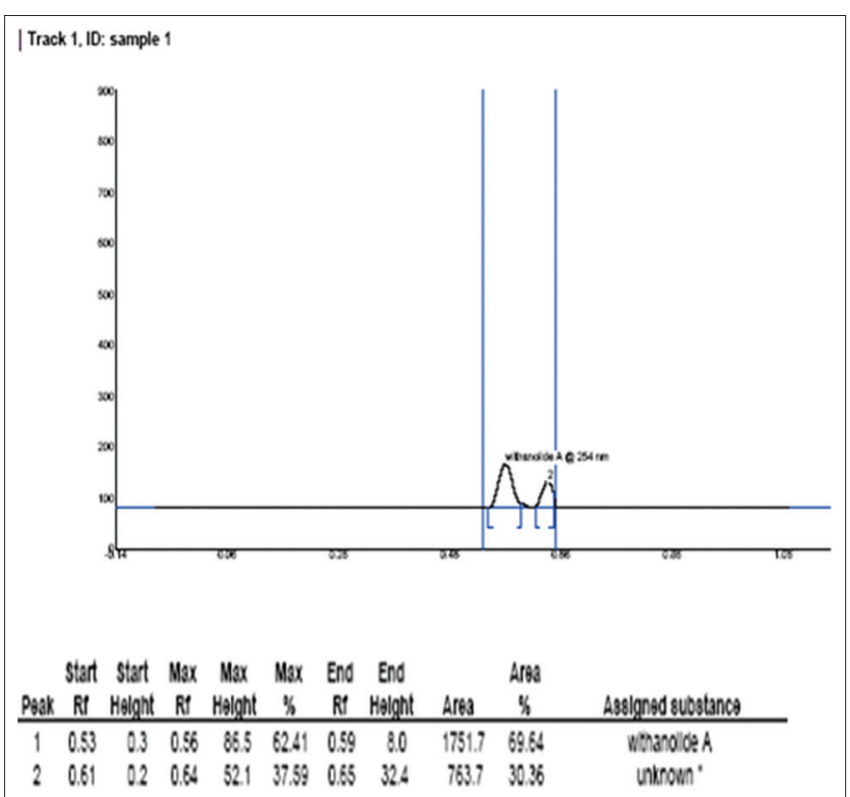

Fig. 2: Withanolide A conc. in sample 1

Detection was done by scanner 3_150306; the optimized optical system was light; D2 lamp; wavelength $254 \mathrm{~nm}$; measurement type remission; measurement mode absorption; detector mode was automatic.

\section{RESULTS AND DISCUSSION}

Chromatographicstudies were done with HPTLC to reveal the concentration of withanolide $\mathrm{A}$, and the results suggest that fresh sample of ashwagandha root extracts contains the highest concentration of Withanolide A as compared to shade drying, sun drying, and hot air oven drying, and in these three drying methods, sun drying is much better than shade drying. However, hot air oven drying at $50^{\circ} \mathrm{C}$ for $12 \mathrm{~h}$ shows the highest yield of Withanolide A than other two (sun and shade) drying methods. Therefore, hot air drying may be the best method of drying for ashwagandha roots.

These results show that 6 days' sun drying is sufficient for roots of ashwagandha. Moreover, moisture content limit for dried ashwagandha root is $6 \%$ or below $6 \%[10]$.

These results show that 15 days' shade drying is sufficient for the roots of ashwagandha. The moisture content limit for dried ashwagandha root is $6 \%$ or below $6 \%$. Moreover, the experimental moisture content for dried roots of Ashwagandha was 5.8\% recorded. 


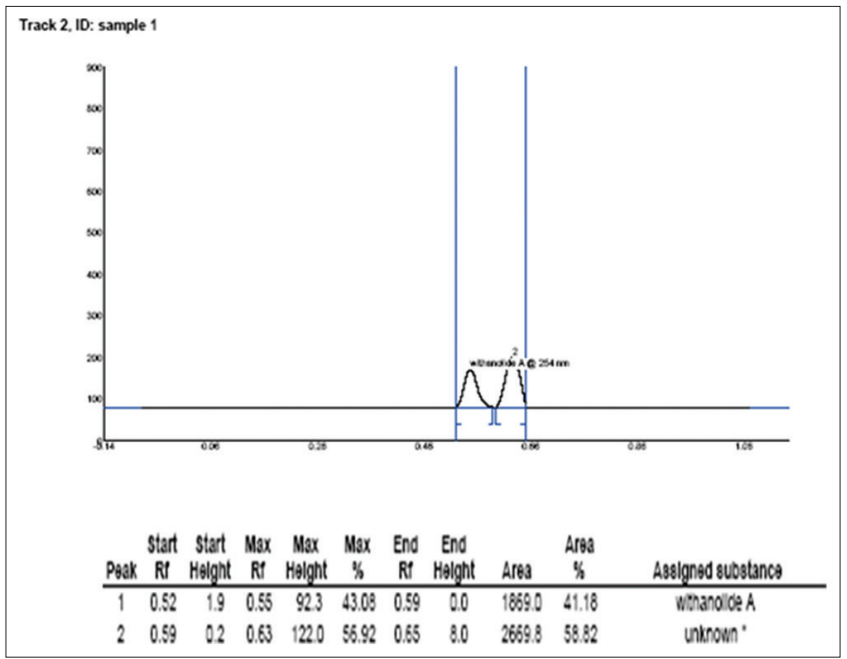

Fig. 3: Withanolide A conc. In sample 1

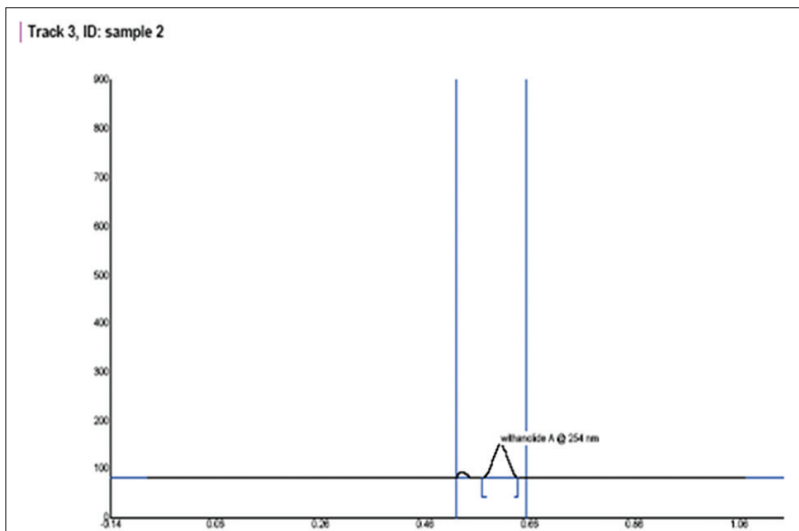

Start Start Max Max Max End End Area Peak Rf Height Rf Height \& Rf Height Ares \&

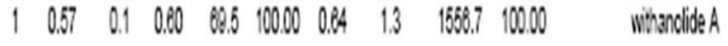

Fig. 4: Withanolide A conc. In sample 2

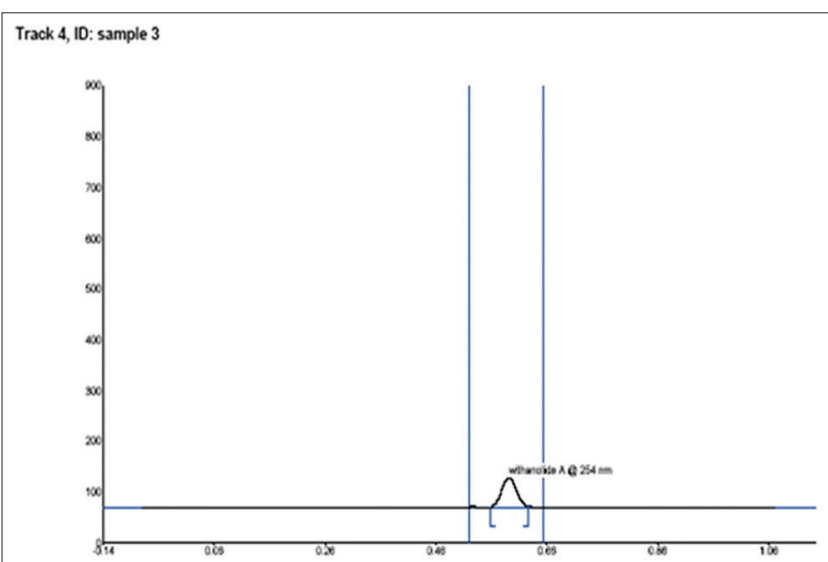

Stat Start Max Max Max End End Ares Peak Rf Height Rf Height It Rif Height Apes \& Assignedsubstance

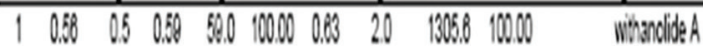

Fig. 5: Withanolide $A$ conc. In sample 3

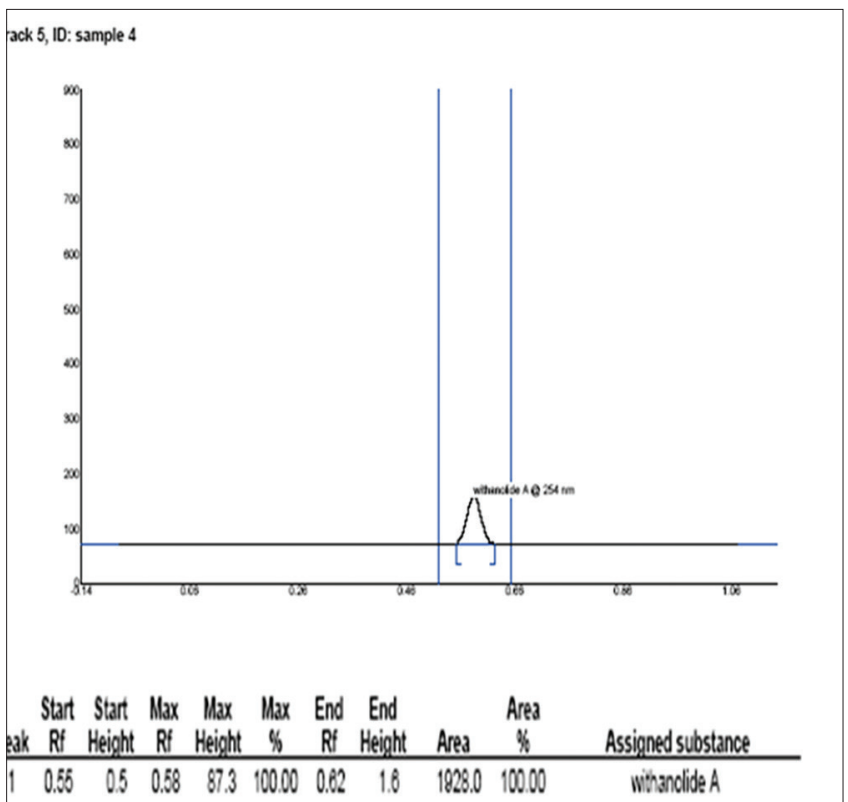

Fig. 6: Withanolide A conc. In sample 4

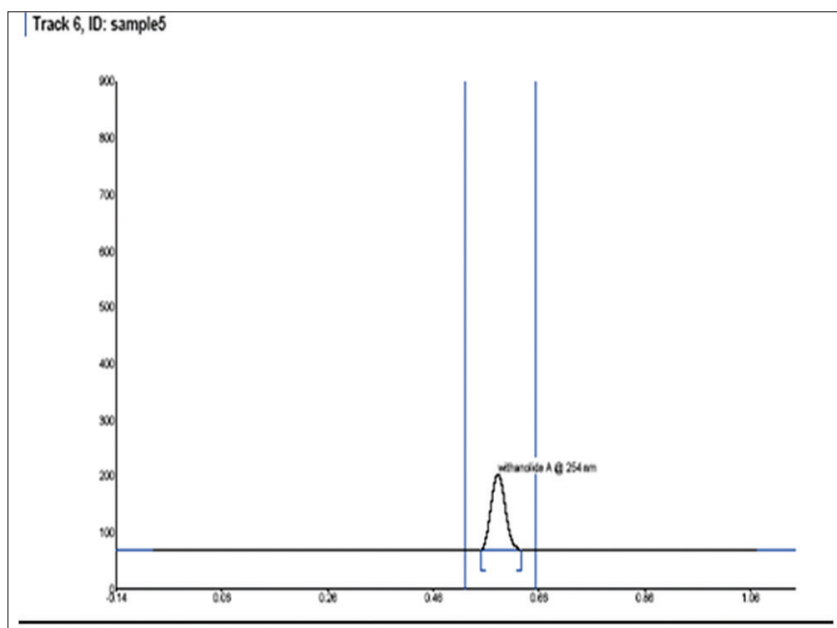

Start Start Max Max Max End End Ares Peak Rf Height Rf Height \& $R$ ff Height Ares \& Assigned substance

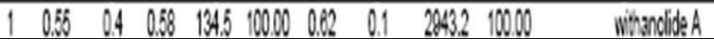

Fig. 7: Withanolide A conc. In sample 5

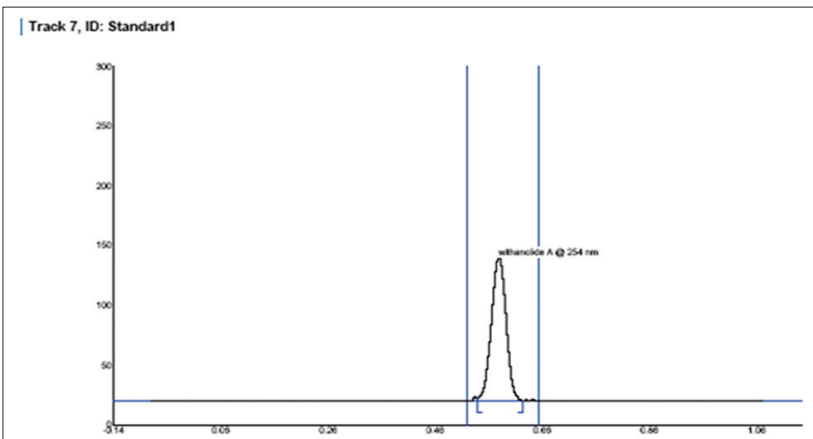

Start Start Max Max Max End End Area Peak Rf Hejght Ri Height \& Ri Height Areg \& Assigned substance $\begin{array}{llllllllllll}1 & 0.54 & 24 & 0.58 & 120.3 & 10000 & 0.82 & 0.1 & 2714.8 & 10000 & \text { witrandide } A\end{array}$

Fig. 8: Withanolide A conc. In standard 1 
Table 7: Comparison between standard and samples for the concentration of Withanolide A (Result through area)

\begin{tabular}{|c|c|c|c|c|}
\hline S. No. & Sample ID & Sample name & Area & Withanolide A (\%) \\
\hline 1. & Sample 1 & Fresh root extract of WS & 1751.72 & 0.03 \\
\hline 2. & Sample 2 & Shade-dried root extract of WS & 1556.70 & 0.009 \\
\hline 3. & Sample 3 & Sun dried root extract of WS & 1305.60 & 0.010 \\
\hline 4. & Sample 4 & $10 \mathrm{~h}$ hot air oven dried at $50^{\circ} \mathrm{C}$ & 1927.95 & 0.014 \\
\hline 5. & Sample 5 & $12 \mathrm{~h}$ hot air oven dried at $50^{\circ} \mathrm{C}$ & 2943.25 & 0.021 \\
\hline 6. & Standard 1 & Withanolide A & 2714.84 & \\
\hline 7. & Sample 6 & $14 \mathrm{~h}$ hot air oven dried at $50^{\circ} \mathrm{C}$ & 1387.94 & 0.010 \\
\hline 9. & Sample 8 & $12 \mathrm{~h}$ hot air oven dried at $60^{\circ} \mathrm{C}$ & 1942.27 & 0.016 \\
\hline 10. & Sample 9 & $14 \mathrm{~h}$ hot air oven dried at $60^{\circ} \mathrm{C}$ & 2193.86 & 0.012 \\
\hline 11. & Sample 10 & $10 \mathrm{~h}$ hot air oven dried at $70^{\circ} \mathrm{C}$ & 1222.68 & 0.008 \\
\hline 12. & Sample 11 & $12 \mathrm{~h}$ hot air oven dried at $70^{\circ} \mathrm{C}$ & 1089.47 & 0.011 \\
\hline 13. & Sample 12 & $14 \mathrm{~h}$ hot air oven dried at $70^{\circ} \mathrm{C}$ & 1141.99 & 0.0031 \\
\hline
\end{tabular}

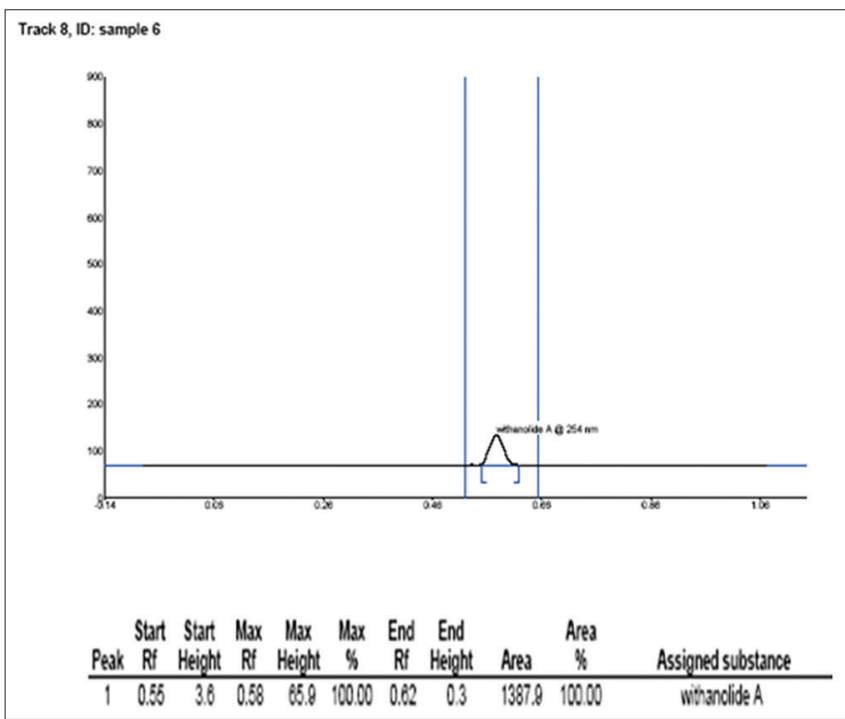

Fig. 9: Withanolide A conc. In sample 6

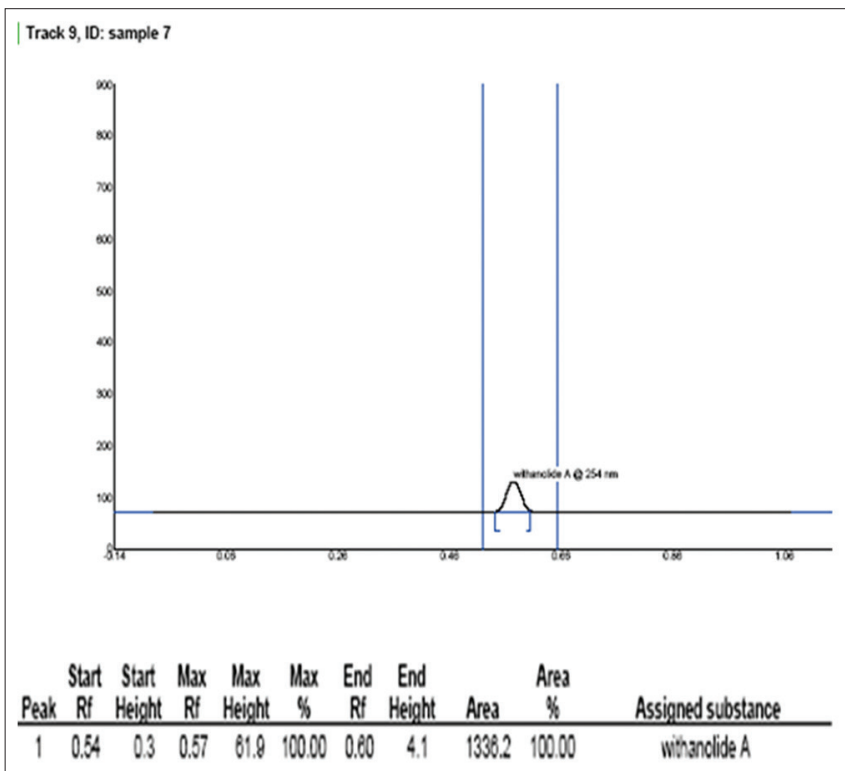

Fig. 10: Withanolide A conc. In sample 7

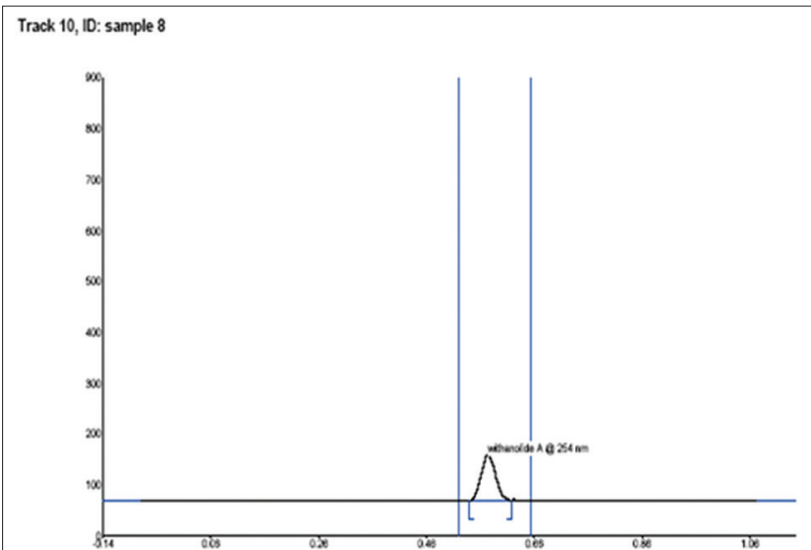

Start Start Max Max Max End End Ares Peak Rf Height $\mathrm{Rf}$ Height \& $\mathrm{Rf}$ Height Ares \& \begin{tabular}{lllllllllll}
\hline 1 & 0.54 & 0.1 & 0.57 & 80.3 & 10000 & 0.82 & 0.7 & 1042.3 & 10000 & whondide A
\end{tabular}

Fig. 11: Withanolide A conc. In sample 8

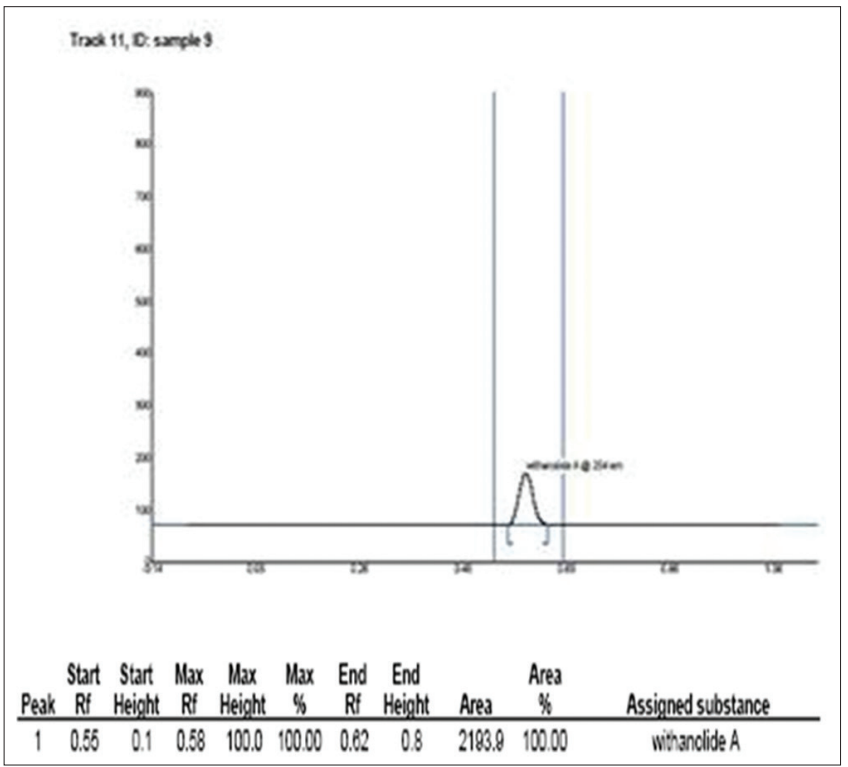

Fig. 12: Withanolide A conc. In sample 9 


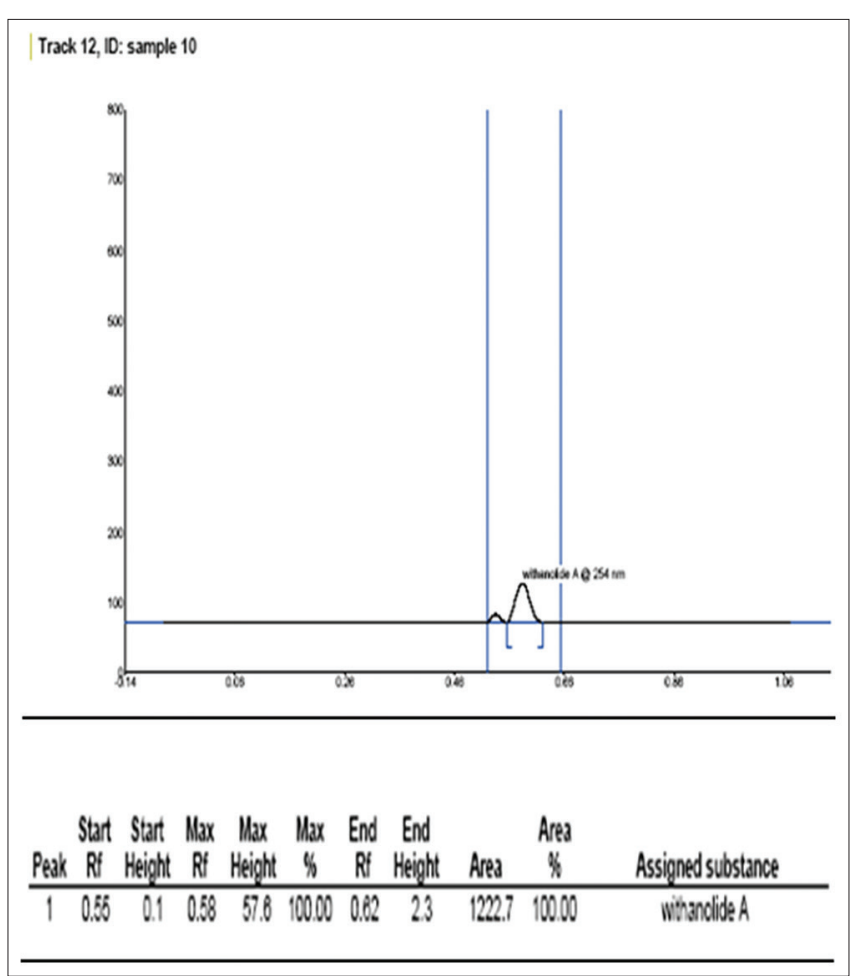

Fig. 13: Withanolide A conc. In sample 10

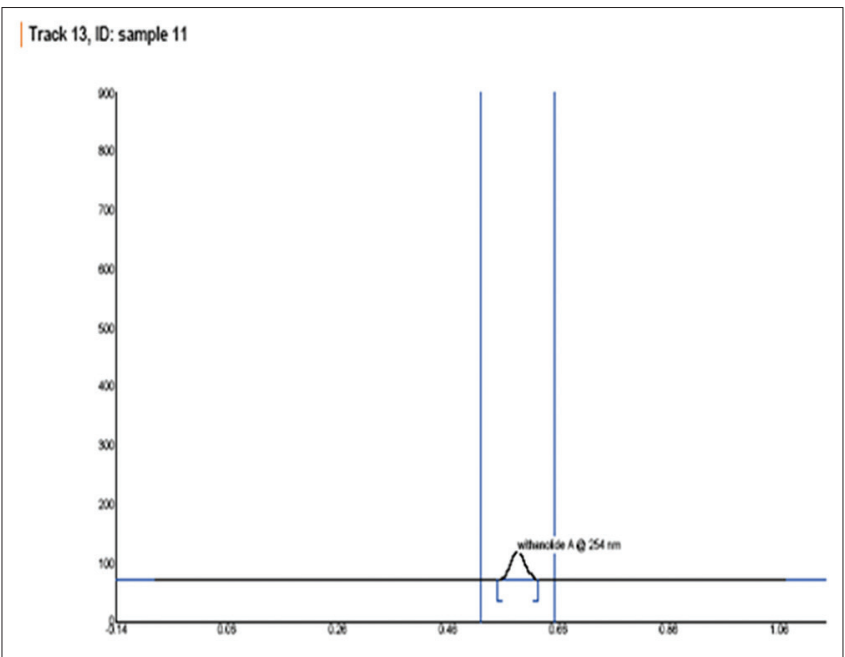

Start Start Max Max Max End End

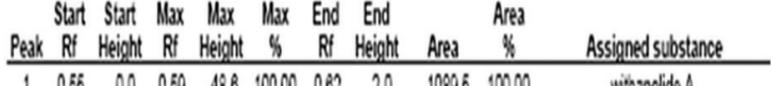

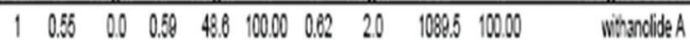

Fig. 14: Withanolide A conc. In sample 11

When roots were kept in hot air oven for $12 \mathrm{~h}$ at $50^{\circ} \mathrm{C}$ and $60^{\circ} \mathrm{C}$ was sufficient for rapid drying of roots of Ashwagandha because it was attaining $6.2 \%$ and $5.8 \%$ moisture content at that time and temperature. Moreover, moisture content limit for dried ashwagandha root is $6 \%$ or below $6 \%$ [10].

In hot air oven, $12 \mathrm{~h}$ at $50^{\circ} \mathrm{C}$ is sufficient for rapid drying of roots of ashwagandha because it attains $6.0 \%$ moisture content at this time and temperature. Moreover, moisture content limit for dried ashwagandha root is $6 \%$ or below $6 \%$ [10].

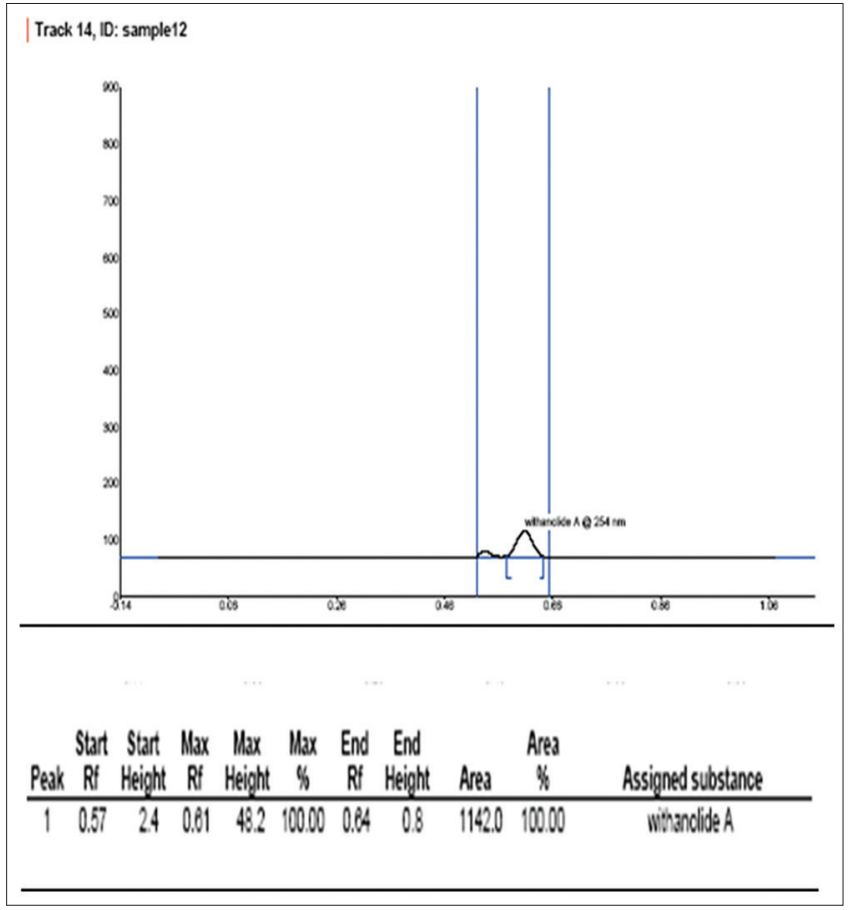

Fig. 15: Withanolide A conc. In sample 12

Hot air oven drying for $12 \mathrm{~h}$ at $60^{\circ} \mathrm{C}$ is sufficient for rapid drying of the roots of ashwagandha because it attains 5.8\% moisture content at this time and temperature. Moreover, moisture content limit for dried ashwagandha root is $6 \%$ or below $6 \%$ [10].

In hot air oven, $10 \mathrm{~h}$ at $70^{\circ} \mathrm{C}$ is sufficient for rapid drying of roots of Ashwagandha because it attains 5.2\% moisture content at this time and temperature. Moreover, moisture content limit for dried ashwagandha root is $6 \%$ or below $6 \%$ [10].

Table 7 shows that chromatographic studies were done with HPTLC to reveal the concentration of Withanolide $\mathrm{A}$, and the results suggest that fresh sample of WS root extracts contains the highest concentration of Withanolide A as compared to shade drying, sun drying and hot air oven drying and in these three drying methods. Sun drying is much better than shade drying. However, hot air oven drying at $50^{\circ} \mathrm{C}$ for $12 \mathrm{~h}$ shows the highest yield of Withanolide A than other two (sun and shade drying) drying methods. Therefore, hot air oven drying may be the best method of drying for WS roots.

\section{CONCLUSION}

On a comparative account, it can be suggested that WS roots may be dried by sun drying or hot air oven drying method. There may be little loss of withanolide A on drying by these two methods. Hence, these two methods of drying may be the safe method for the drying of ashwagandha roots.

\section{CONFLICTS OF INTEREST}

The authors have no conflicts of interest.

\section{AUTHORS' CONTRIBUTIONS}

All authors contributed to the design and implementation of the research, to the analysis of the results, and to the writing of the manuscript.

\section{REFERENCES}

1. Desilva T. Chapter-industrial utilization of medicinal plants in developing countries. Book of Medicinal Plants for Forest Conservation and Health Care. Rome, Italy: Food and Agriculture Organization of the 
United Nations; 2005

2. Akowuah GA, Zhari I, Norhayati I, Maram A. HPLC and HPTLC densitometric determination of andrographolides and antioxidant potential of Andrographis paniculata. J Food Compost Anal 2006; 19:118-26.

3. Bhan MK, Dhar AK, Khan S, Lattoo SK, Gupta KK, Choudhary DK, Screening and optimization of Andrographis paniculata (Burm.f.) nees for total andrographolide content, yield and its components. Sci Hortic 2006;107:386-91.

4. Alwakeel SS, Microbial and heavy metal contamination in herbal medicines. Res J Microbiol 2008;3:683-91.

5. Misra L, Mishra P, Pandey A, Sangwan RS, Sangwan NS, Tuli R, et al. Withanolides from Withania somnifera roots. Phytochemistry 2008;69:1000-4.

6. Alwadi HM, Baka ZA. Microorganisms asssociated with Withania somnifera leaves. Microbiol Res 2001;156:303-9.

7. Jose KP and Joy CM, Postharvest processing of spices in relation to export quality. Everyman's Sci 2004;39:230-5.

8. Bachmann J, Earles R. Postharvest Handling of Fruits and Vegetables. Washington, D.C: NCAT agriculture specialists published, ATTRA Publication \#IP116; 2000

9. Kulkarni SK, Dhir A. Withania somnifera: An Indian ginseng. Prog
Neuropsychopharmacol Biol Psychiatry 2008;32:1093-105.

10. Evans WC, Pharmacognosy. Noida, U P, India; Published by Elsevier (India) Pvt Ltd.; 2007. p. 15, 64.

11. Anonymous. Available from: http://www.del.icio.us/dig/reddit/furl. com. How to Dry Fruits and Vegetables. 2009.

12. Khan TA, Gohell AK, Mallya R. Svkm's standardisation and HPTLC method development of marketed ayurvedic formulation-balarishta. Int J Pharm Pharm Sci 2016;8.

13. Jena S, Jena RC, Bhol R, Agarwal K, Sarangi A, Sahu PK, Mishra S. A comparative study of the anti-oxidative and anti-diabetic potential of in vitro and in vivo root and leaf extracts of Withania somnifera on streptozotocin induced diabetic rats. Int J Pharm Pharm Sci 2016;8.

14. Elgar J. Hort Research. Mt Albert, Hort FACT-Carnations-Harvesting and Post-harvest Practices for Commercial Production, vol. 6, 1998. Anonymous. Available from: http://www.hortnet.co.nz/copy.html

15. Rashmin. An introduction to analytical method development for pharmaceutical formulations. 2008. Anonymous. Available from: http://www.pharmainfo.net.

16. Araya OS, Ford JH, St Wort J (1981) Reported Hypericum perforatum is a Perennial Herbaceous Plant, Air Drying of the Level of Hypericin by up to $80 \%$ Most Likely as a Result of Oxidation. (United states patent 6128831), Process for Drying Medicinal Plants, 1999. 